\title{
CONSTRUCTIONS ON LOW-DIMENSIONAL DIFFERENTIABLE MANIFOLDS
}

\author{
BY VALENTIN POÉNARU ${ }^{1}$
}

Communicated by Wllliam Browder, May 29, 1967

1. This note contains the statements of three theorems on lowdimensional differentiable manifolds (dimensions 3 and 4 ). The proofs, which use techniques partly connected to [1], will appear elsewhere.

We denote by $M_{n}+\left(\phi_{\lambda}\right)$ the manifold obtained by adding the handle of index $\lambda, D_{\lambda} \times D_{n-\lambda}$, via the embedding $\phi_{\lambda}: S_{\lambda-1} \times D_{n-\lambda}$ $\rightarrow \partial M_{n}$, to $M_{n}$. More generally, we shall use the following notation: If $P_{n-1} \subset S_{n-1}=\partial D_{n}$ is a bounded submanifold and $\psi: P_{n-1} \rightarrow M_{n}$ an embedding, we denote by $M_{n}+(\psi)$, the space $M_{n} \cup D_{n}$, where every $x \in P_{n-1}$ is identified to $\psi(x) \in M_{n}$. It is understood that, if $\psi\left(P_{n-1}\right)$ $C \partial M_{n}$, then $M_{n}+(\psi)$ is a "usual" differentiable manifold, otherwise a "singular" one (see $\$ 2)$.

Theorem 1. Let $M_{3}$ be a compact, differentiable, homotopy 3-disk. Then $M_{3} \times I$ is diffeomorphic to $D_{4}$ with handles of index 2 and 3 added:

$$
M_{3} \times I=D_{4}+\left(\phi_{2}^{1}\right)+\cdots+\left(\phi_{2}^{p}\right)+\left(\phi_{3}^{1}\right)+\cdots+\left(\phi_{3}^{p}\right) .
$$

Hence, one can eliminate the handles of index 1 of $M_{3} \times I$ (compare with the similar procedure, in higher dimensions [2]).

In fact we obtain Theorem 1 from the slightly stronger:

TheOREM $1^{\prime}$. If $M_{3}$ is a compact, differentiable homotopy 3-disk, there exists an integer $p=p\left(M_{3}\right)$ such that:

$$
\begin{gathered}
\left(M_{3} \#\left(S_{2} \times I\right) \# \cdots \#\left(S_{2} \times I\right)(p \text { times })\right) \times I \\
=D_{4}+\left(\phi_{2}^{1}\right)+\cdots+\left(\phi_{2}^{p}\right) .
\end{gathered}
$$

This together with some immersion theory, implies easily the main result from [1].

The next theorem is the main step in proving Theorem $1^{\prime}$. But in order to state it, we need some preparation.

2. We consider the 3-manifold $T_{p}=\left(S_{1} \times D_{2}\right) \#\left(S_{1} \times D_{2}\right) \# \cdots$ $\#\left(S_{1} \times D_{2}\right) \quad(p$ times $)$ and its double $2 T_{p}=\left(S_{1} \times S_{2}\right) \#\left(S_{1} \times S_{2}\right)$ $\#$ \#...\# $\left(S_{1} \times S_{2}\right)$ ( $p$ times). (\# means connected sum.) A family of $p$ 2-by-2 disjoint embeddings $\phi_{i}: S_{1} \rightarrow T_{p}(i=1, \cdots, p)$ is called

1 This research has been supported by NSF Grant GP-6299. 
"unknotted," if, after a diffeomorphism of $T_{p}$, the images become $\left(S_{1} \times 0\right) \cup\left(S_{1} \times 0\right) \cup \ldots \cup\left(S_{1} \times 0\right)$ ( $p$ times, $0=$ center of $\left.D_{2}\right)$. Similarly, if $o \in S_{2}$, is some fixed point, a family of $p, 2$-by-2 disjoint embeddings $\psi_{i}: S_{1} \rightarrow 2 T_{p}(i=1, \cdots, p)$ is called "unknotted" if after a diffeomorphism of $2 T_{p}$, the images become $\left(S_{1} \times 0\right) \cup \ldots$ $\cup\left(S_{1} \times 0\right)$ ( $p$ times). (The difference between the two notions is illustrated by the Figures 1a, 1b, where "knotted" embeddings $S_{1} \rightarrow T_{1}$ are presented, such that the composite embeddings $S_{1} \rightarrow T_{1}$ $\subset 2 T_{1}$ are unknotted. Figure $1 \mathrm{~b}$ contradicts, unfortunately, the obvious conjecture suggested by Figure 1a.)

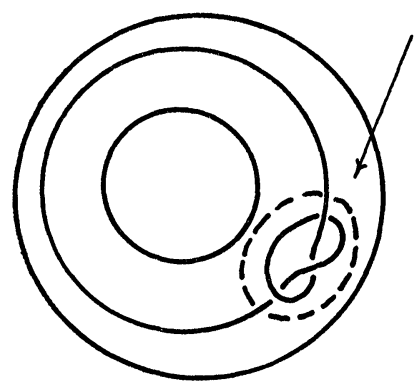

Figure 1a

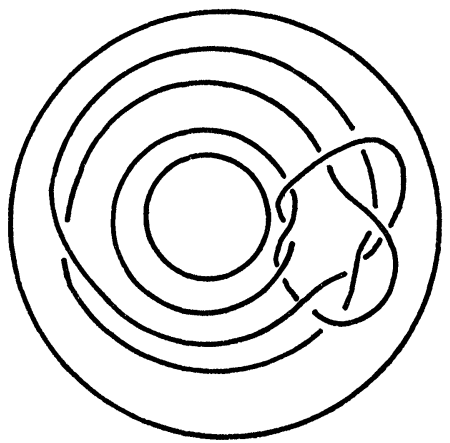

Figure 1b

We consider now (compact) 3-manifolds with singularities. These will be compact spaces $V_{3}$ which are everywhere (bounded) differentiable manifolds, except for a finite number of compact neighborhoods $W$, which admit descriptions of the following type: We consider two embeddings $\phi, \psi: I \rightarrow S_{2}=\partial D_{3}$, such that $\phi(I) \cap \psi(I)$ consists of exactly one point, with transversal intersection, and two thin tubular neighborhoods around them: $\Phi, \Psi: I \times I \rightarrow S_{2}=\partial D_{3} . I \times I$ is assimilated to $I \times I \times o \subset \partial(I \times I \times I)=\partial D_{3}$ and then $W$ is our original $D_{3}$ (target of $\phi, \psi$ ) with two other copies of $D_{3}$ added along $\Phi, \Psi$ :

$$
W=D_{3}+(\Phi)+(\Psi)=D_{3}+(\Psi)+(\Phi) \text {. }
$$

$V_{3}$ is "regular" except for a "singular" set $\sigma\left(V_{3}\right)$ which is a bounded 2-manifold, having as connected components various copies of $D_{2}$.

We consider resolutions (of singularities) for $V_{3}, \Pi: V_{3}^{\prime} \rightarrow V_{3}$ where $V_{3}^{\prime}$ is a nonsingular 3-manifold, $\Pi^{-1}(x)$ has exactly 2 elements if $x \in$ int $\sigma\left(V_{3}\right)$ and exactly 1 element if $x$ is regular. (If $x \in \partial \sigma\left(V_{3}\right)$, as we shall see in a moment, $\Pi^{-1}(x)$ has one point in half the cases and two in the other half.) It is moreover understood that, if $W$ is 
as before, $\Pi^{-1}(W)=W^{\prime}$ is obtained by cutting the $I \times I \times I$ corresponding to $\Phi$, from $D_{3}+(\Psi)$ along $\Phi^{-1}$ (Image $\Phi \cap$ Image $\Psi$ ) (or the $I \times I \times I$ corresponding to $\Psi$, from $\left.D_{3}+(\Phi) \cdots\right)$. So $W^{\prime}=S_{1} \times D_{2}$, and passing from $W$ to $W^{\prime}$, Image $\Phi \cap$ Image $\Psi=I \times I$ "blows up" into $\beta=\Phi^{-1}($ Image $\Phi \cap$ Image $\Psi)+\Phi \Phi^{-1}($ Image $\Phi \cap$ Image $\Psi$ ), diffeomorphic to $S_{1} \times I$ (the first summand is in $I \times I \times I$, the other in $D_{3}+(\Psi)$ ). We say that $\Phi$ (or $\Psi$ ) is specified in the resolution $\Pi=V_{3}^{\prime}$ $\rightarrow V_{3}$. (One remarks that the two $I \times I \times I=D_{\mathbf{3}}$ play a symmetric role in $W$, but cannot be interchanged with the original $D_{3}$; this is easily seen by looking at the sheaf of local homology groups along $\sigma(W)=$ Image $\Phi \cap$ Image $\Psi$.)

If $\Phi$ is specified in the resolution $\Pi: V_{3}^{\prime} \rightarrow V_{3}$, as above, there exists a canonical embedding $j: W^{\prime} \rightarrow S_{3}$ which is uniquely determined (up to isotopy) by the requirements that $j\left(W^{\prime}\right)$ be unknotted and $j(\beta)$ be contained in a nonsingular 2-disk of $S_{3}$.

Let us consider the category $R$ of resolutions $\Pi: V_{3}^{\prime} \rightarrow V_{3}$ (for all $V_{3}$ 's) where the morphisms are given by commutative squares, having $\Pi$ on the verticals and embeddings on the horizontals. Let us also consider the category $\mathcal{C}$ consisting of triples $\left(M_{4}, j, M_{3}\right)$ where $M_{4}$ is a bounded differentiable 4-manifold, $M_{3}$ a (bounded) differentiable 3-manifold and $j: M_{3} \rightarrow \partial M_{4}$ an embedding. Morphisms are again commutative squares having the $j$ 's on the verticals and embeddings on the horizontals. We have:

Lemma. There exists a unique ("thickening") functor $\Theta: R \rightarrow \mathcal{C}$ such that, if $\eta \in \mathbb{R}$ is $\Pi: V_{3}^{\prime} \rightarrow V_{3}$ then $\Theta(\eta)=\left(\Theta_{4}(\eta), j(\eta), V_{3}^{\prime}\right)$ and the following requirements are fulfilled:

(a) If $V_{3}$ is nonsingular $\left(\sigma\left(V_{3}\right)=\varnothing\right)$ and $\eta$ is the (only possible) resolution: identity: $V_{3} \rightarrow V_{3}$, then $\Theta_{4}(\eta)=V_{3} \times I$ and $j(\eta)$ is $V_{3} \times 0$ $C \partial\left(V_{3} \times I\right)=V_{3} \times o+\partial V_{3} \times I+V_{3} \times 1$.

(b) $\Theta$ is compatible with the connected sum \# and, more generally, let $V_{3}={ }_{0} V_{3}+{ }_{1} V_{3}$, with $V_{3} \cap_{1} V_{3}=M_{2} \subset V_{3}-\sigma\left(V_{3}\right)$ (a compact 2-manifold $)$. If $\eta=\left(\Pi: V_{3}^{\prime} \rightarrow V_{3}\right)$ is a resolution for $V_{3}, M_{2}$ can be lifted to a unique $M_{2}^{\prime} \subset V_{3}^{\prime}$, and $\eta$ can be restricted to resolutions o $\eta$ and ${ }_{1} \eta$. By (a) and the functoriality of $\Theta$ there exist well-defined embeddings $M_{2}^{\prime} \times I$ $C \partial \Theta_{4}\left({ }_{o} \eta\right)$ and $M_{2}^{\prime} \times I \subset \partial \Theta_{4}\left({ }_{1} \eta\right)$ (coming from the corresponding $j$ 's). $\Theta_{4}(\eta)$ is obtained by pasting $\Theta_{4}(0 \eta)$ and $\Theta_{4}\left({ }_{1} \eta\right)$ together along $M_{2} \times I$, and $j(\eta)$ in a similar way from $j\left({ }_{\circ} \eta\right), j\left({ }_{1} \eta\right)$.

(c) $\Theta\left(W^{\prime} \stackrel{M}{\rightarrow} W\right)=\left(D_{4}, j: W^{\prime} \rightarrow S_{3}=\partial D_{4}, W^{\prime}\right)$ where $W^{\prime}, W$ are as above, and $j$ is the canonical embedding. (It is understood that $\Theta(\eta)$ is determined only up to "isomorphism.")

This lemma is implicit in [1]. 
3. We are interested in 3-manifolds with singularities $V_{3}$, which admit the following description:

We consider $T_{2 p}$ and $2 p$ differentiable embeddings $\phi^{i}: S_{1} \rightarrow T_{2 p}$ $(i=1, \cdots, 2 p)$ such that $\phi^{i}\left(S_{1}\right) \cap \phi^{j}\left(S_{1}\right)=\varnothing$ except for $\phi^{2 k-1}\left(S_{1}\right)$ $\bigcap \phi^{2 k}\left(S_{1}\right)$ which consists of exactly 2 points, with transversal intersection $(k=1, \cdots, p)$.

We remark that $\phi^{2 k-1}\left(S_{1}\right) \cup \phi^{2 k}\left(S_{1}\right)$ contains exactly 4 simple circuits of $\partial T_{2 p}$ and, for each $k=1, \cdots, p$, we consider a differentiable embedding $\psi^{k}: S \rightarrow \partial T_{2 p}-U_{1}^{2 k} \phi^{i}\left(S_{1}\right)$, "parallel" to one of these 4 circuits. We assume that $\psi^{i}\left(S_{1}\right) \cap \psi^{i}\left(S_{1}\right)=\varnothing$. We consider some very thin tubular neighborhoods: $\Phi^{i}, \Psi^{j}: S_{1} \times I \rightarrow \partial T_{2 p}(i=1, \cdots, 2 p$; $j=1, \cdots, p)$ of $\phi^{i}, \psi^{j} . S_{1} \times I$ is assimilated to $\left(\partial D_{2}\right) \times I \subset \partial\left(D_{2} \times I\right)$, and hence we can add $3 p$ times $D_{2} \times I$ along the $\Phi$ and $\Psi$ 's, to $T_{2 p}$. We get in this way a 3-manifold with singularities

$$
V_{3}=T_{2 p}+\left(\Phi^{1}\right)+\cdots+\left(\Phi^{2 p}\right)+\left(\Psi^{1}\right)+\cdots+\left(\Psi^{p}\right) .
$$

We shall consider a resolution $\Pi: V_{3}^{\prime} \rightarrow V_{3}$ which specifies $\Phi^{2}$, $\Phi^{4}, \cdots, \Phi^{2 p}$.

We shall also consider, for each $k=1, \cdots, p$ an embedding $\phi^{2 k-1}: S_{1} \rightarrow$ int $T_{2 p}$, very close to $\phi^{2 k-1}$, and "parallel" to it.

Finally we denote by $\bar{T}_{2 p}$ the 3-manifold:

$$
\bar{T}_{2 p}=T_{2 p}+\left(\partial T_{2 p}\right) \times I \quad \text { where } \partial T_{2 p}=\partial T_{2 p} \times o .
$$

With this we can state

TheOREM 2. Let $M_{3}$ be a compact homotopy 3-disk. Then, for some $p=p\left(M_{3}\right)$, the differentiable manifold:

$$
\begin{aligned}
M^{p} & =\left(M_{3} \#\left(S_{2} \times I\right) \# \cdots \#\left(S_{2} \times I\right)(p \text { times })\right) \times I \\
& =\left(M_{3} \times I\right) \#\left(S_{2} \times D_{2}\right) \# \cdots \#\left(S_{2} \times D_{2}\right)(p \text { times })
\end{aligned}
$$

can be described as follows:

There exists a $V_{3}$ as above, for which the following requirement is fulfilled:

( $\gamma$ ) The $2 p$ embeddings $S_{1} \rightarrow 2 \bar{T}_{2 p}$ :

$$
S_{1} \stackrel{\phi^{2 k}, \Phi^{2 k-1}}{\longrightarrow} T_{p} \subset \bar{T}_{2 p} \subset 2 T_{2 p} \quad(k=1, \cdots, p)
$$

are unknotted.

Moreover,

$$
\Theta_{4}\left(\Pi: V_{3}^{\prime} \rightarrow V_{3}\right)=M_{4}^{p} \quad \text { (diffeomorphism). }
$$


One remarks that the statement $M_{3}=D_{3}$ is equivalent to $M_{4}^{p}=D_{4}$ $\#\left(S_{2} \times D_{2}\right) \# \cdots \#\left(S_{1} \times D_{2}\right)$ ( $p$ times) (see [1]). This motivates

TheOREM 3. Let $V_{3}$ be the singular 3-manifold described above, but such that the requirement $(\gamma)$ is replaced by the stronger requirement $(\Gamma)$ :

( $\Gamma)$ The $2 p$ embeddings $S_{1} \rightarrow \bar{T}_{2 p}$ :

$$
S_{1} \stackrel{\phi^{2 k}, \phi^{2 k-1}}{\longrightarrow} T_{2 p} \subset \bar{T}_{2 p} \quad(k=1, \cdots, p)
$$

are unknotted.

Then:

$\Theta_{4}\left(\Pi: V_{8}^{\prime} \rightarrow V_{8}\right)=D_{4} \#\left(S_{2} \times D_{2}\right) \# \cdots \#\left(S_{2} \times D_{2}\right)(p$ times $)$

(diffeomorphism).

\section{BIBLIOGRAPHY}

1. V. Poenaru, Sur les varietés tridimensionnelles ayant le type d'homotopie de $S_{8}$, Séminaire Ehresmann 6 (1963), 1-67.

2. S. Smale, Generalized Poincare conjecture in dimensions >4, Ann. of Math. 74 (1961), 391-406.

NORTHEASTERN UNIVERSITY 